Veritas E Scientia

Vol. 8. $\mathrm{N}^{\circ} 1$.

Enero - Junio del 2019.

ISSN Edición Online: 2617-0639

https://doi.org/10.47796/ves.v8i1.115

\title{
RELACIÓN ENTRE LA PARTICIPACIÓN EN FERIAS COMERCIALES, Y EL DESEMPEÑO DE LAS EMPRESAS ASOCIADAS EN PRO OLIVO 2016 - 2017"
}

\author{
RELATIONSHIP BETWEEN PARTICIPATION IN COMMERCIAL FAIRS, AND THE PERFORMANCE OF \\ ASSOCIATED COMPANIES IN PRO OLIVO 2016 - 2017
}

Lucio Walter M. Valderrama Pérez ${ }^{1}$

Juan Guillermo Aranibar Ocola²

Presentado: $19 / 12 / 2018$

Aceptado: 19/04/2019

Publicado online:17/07/2019

\begin{abstract}
RESUMEN
El desarrollo del trabajo de investigación estuvo enfocado a la relación que existe entre la participación en ferias comerciales de las empresas asociadas en Pro Olivo y su desempeño, periodo 2016 - 2017, lo que permite establecer cómo la participación en ferias comerciales se relaciona con el desempeño, promoción del producto, nivel competitivo y el logro de objetivos empresariales; el presente trabajo de investigación es no experimental de nivel explicativa, porque se obtienen datos directamente de la realidad objeto de estudio de acuerdo a la finalidad de la investigación. El área de investigación está enmarcada en el comercio exterior, relacionado a las ferias internacionales en el departamento de Tacna considerada como una región que tiene vocación exportadora desarrollada las misma que son impulsadas por los organismos estatales y principalmente por el Ministerio de la Producción quien viene implementando políticas públicas de promoción de exportaciones. Así mismo se viene observando que falta mejorar el desempeño de las empresas asociadas a Pro Olivo, lo que va a permitir mejorar su productividad, la calidad de sus productos y por lo tanto tengan una mejor oferta frente a los mercados, internacionales. Se concluye que existe relación directa entre la participación en ferias comerciales de las empresas asociadas en Pro Olivo y su desempeño.
\end{abstract}

Palabras Clave: Ferias comerciales, desempeño empresarial.

\section{ABSTRACT}

The development of this research work is focused on the relationship between the participation in trade fairs of the associated companies in Pro Olivo and its performance, period 2016 - 2017, which allows to establish how participation in trade fairs is related to performance, product promotion, competitive level and achievement of business objectives; The

\footnotetext{
${ }^{1}$ Magíster en Ingeniería Comercial y Negocios Internacionales

2 Doctor, Jefe de la Oficina de Contabilidad e la Universidad Privada de Tacna
} 
present research work is non-experimental at an explanatory, descriptive level, because data is obtained directly from the reality object of study according to the purpose of "the research. The area of "research is framed in foreign trade, related to international fairs in the department of Tacna considered as a region that has a developed export vocation that are driven by state agencies and mainly by the Ministry of Production who comes implementing public policies to promote "exports. Likewise, "it has been observed that there is a need to improve the performance of the companies associated with Pro Olivo, which will allow improving their productivity, the quality of their products and therefore have a better offer in front of the" international markets. It is concluded that there is a direct relationship between the participation in trade fairs of the associated companies in Pro Olivo and their "performance.

Keywords: Trade fairs, business performance.

\section{INTRODUCCIÓN}

Tacna viene siendo una región que tiene vocación exportadora desarrollando la implementación de políticas públicas para la promoción de exportaciones a un nivel agresivo e importante, son muchos los programas que el ministerio de la Producción viene implementado actualmente con un nivel de presupuesto interesante, que incluye la participación en ferias, pasantías, misiones técnicas comerciales, ruedas de negocios y financiamiento de proyectos de innovación entre otros muchos e importantes. El desempeño de las empresas asociadas a Pro Olivo, hacen que mejoren su capacidad productiva, la calidad de sus productos y por lo tanto tengan una mejor oferta y participación en los diferentes mercados internacionales. Actualmente, observamos un contexto de implementación de políticas públicas para la promoción de exportaciones a un nivel agresivo e importante, son muchos los programas que el ministerio de la Producción viene implementado actualmente con un nivel de presupuesto interesante, que incluye la participación en ferias, comerciales, ruedas de negocios y financiamiento de proyectos de innovación entre otros muchos e importantes. Este escenario de promoción de exportaciones actual es muy distinto de cualquier otro que se haya visto con anticipación en el país. En este nuevo entorno de promoción de actividades exportadoras se desenvuelve Pro Olivo, que es una organización sin fines de lucro, que asocia a los procesadores y exportadores de aceitunas, aceite de olivo y otros derivados del olivo. El desempeño de las empresas a Pro Olivo, hacen que mejoren su productividad, la calidad de sus productos y por lo tanto tengan una mejor oferta frente a los mercados, principalmente aquellos que internacionalmente son vecinos nuestros. Pro Olivo, como organización, ya ha sido beneficiario de apoyo técnico y económico para participar de acciones de promoción de exportaciones, la primera de ellas se hizo con la participación en la feria Expo alimentaria en Lima hace tres años donde se expuso de manera conjunta los productos de los asociados, asimismo se ha desarrollado misiones técnicas y comerciales específicas a países muy lejanos como España, Australia, Grecia etc. Se considera que el objetivo de estas acciones de promoción son las de identificar y sobretodo aprovechar las nuevas oportunidades comerciales que se hayan podido determinar. Por ello es importante estudiar cuáles han sido los resultados de participar en ferias y misiones técnicas - comerciales como parte de una política clara de promoción de exportaciones. Es necesario que las pequeñas y medianas empresas participen en ferias comerciales, para que estas descubran sus fortalezas y debilidades, asimismo las ferias permiten conectarse a clientes potenciales en cantidad, también permite posicionar los productos e imagen de la empresa, además es posible relacionarse con nuevos proveedores, todo ello conlleva al incremento de ventas de la empresa.

Niño L. (2014), concluye que las ferias y exposiciones son reconocidas como escenarios en donde empresas y personas interactúan buscando satisfacer necesidades de negocio, conocimiento, esparcimiento, comunicación e interacción, mediante el intercambio de bienes, servicios e 
información. Olivares P. (2014), afirma las ferias internacionales son una herramienta de promoción ampliamente utilizada alrededor del mundo. Mauricio J. (2010), afirma que la adopción de la participación en las ferias como estrategia de comercio y promoción de exportaciones, es función directa del empresario exportador. Estrada (2010) concluye que las ferias Internacionales son un instrumento para mejorar la comunicación. Sirven para conocer las tendencias del mercado y la evolución de los mismos. Según Reyes (2009) también refiere que las ferias son herramientas comerciales que ayudan en el intercambio de ideas y productos, permiten darlos a conocer y de esta manera establecer nuevos contactos, realizar el proceso del cierre de ventas, darse a conocer en el mercado y tener contacto con los medios de comunicación. Angles (2016) hace una evaluación de cómo los expositores relacionan su desempeño con su participación en ferias internacionales realizadas en nuestro país llegando a la conclusión principal que son las ventas el primer factor para evaluar el desempeño de la participación en una feria. Gaviño (2015) indica que las ferias representan una importante fuente de empleo para la sociedad además son una opción para el trabajo de muchos artesanos de la zona. Angles (2016) refiere que la dimensión ventas se relaciona de manera positiva con el desempeño ferial de las mismas. Sarmiento M, (1995) publicó que el origen de las ferias se pierde en los tiempos históricos, y si organiza adecuadamente el contacto de la oferta y la demanda. Mientras las exposiciones buscan objetivos de promoción cultural, social, científico, técnico, turismo, etc., las ferias persiguen objetivos fundamentalmente comerciales . (Sarmiento García, 1995). Las ferias comerciales son un instrumento al servicio del comercio que favorecen el desarrollo económico y facilitan el cumplimiento de los objetivos empresariales de los expositores, de los visitantes y de las organizaciones feriales. (Puchalt J. Munuera, 2008). Las ferias ofrecen una excelente oportunidad para el contacto cara a cara con múltiples colectivos (Godar y O'Connor, 2001; Navarro, 2001; Munuera y Ruiz, 1999). Participar en una feria comercial es una de las mejores estrategias de marketing para una empresa (Peña C, 2017). Jerome McCarthy (padre de las 4 Ps) definió a la promoción como: una serie de actividades cuyo objetivo es: informar, persuadir y recordar las características, ventajas y beneficios del producto. Una promoción es parte de las comunicaciones de marketing y es el medio por el cual una empresa intenta informar, convencer y recordar, directa o indirectamente, sus productos y marcas al gran público (Kotler y Keller, 2012).

Los factores a considerar en la distribución de productos son el tamaño de la orden, nivel de inventario y tiempo de entrega. La distribución debe de considerar la entrega correcta del producto, en el lugar adecuado y en el momento oportuno, con el menor costo posible. (Kotler y Armstrong, 2013). El canal incluye siempre al productor y al cliente final del producto en su forma presente, así como a cualesquiera intermediarios, como los detallistas y mayoristas (Stanton W, 2003). Es la ruta por la que circula el flujo de productos desde su creación en el origen hasta llegar a su consumo o uso en el destino final. (Santesmases M, 2003). Una serie de organizaciones interdependientes facilitan la trasferencia de la propiedad sobre los productos conforme estos se mueven del fabricante hasta el usuario de negocios o el consumidor. (Lamb, Hair, McDanie, 2006). Los canales de comercialización pueden ser considerados como conjuntos de organizaciones interdependientes que intervienen en el proceso por el cual un producto o servicio está disponible para el consumo. (Stern, Louis, 2015). Velázquez E. (2012), dice que la importancia del canal de marketing o de distribución radica en el beneficio que se brinda a los consumidores en cuanto al ahorro tiempo cuando hay que recorrer grandes distancias para satisfacer necesidades mediante un producto o servicio.

El atributo más utilizado en el Marketing, define que el consumidor al momento de adquirir un producto depende de su clase social, cultura y percepción de (Reales H, 2003).

"Las negociaciones se pueden definir prácticamente como el proceso que les ofrece a los contendientes la oportunidad de intercambiar promesas y contraer compromisos formales, tratando de resolver sus diferencias." (Colosi y Berkely, 1981). "La negociación es un proceso y una técnica mediante los cuales dos o más partes construyen un acuerdo" (Monsalve, 1988). "...buscando el mejor resultado posible, de tal manera que todas las partes sean beneficiadas." (Correa y Navarrete, 1997). (Hernández M. (2006), indica que el conocimiento pleno del tipo de proceso negociador resulta de 
vital importancia para su adecuada preparación. La competitividad es la capacidad para desarrollar y mantener unas ventajas comparativas (Pérez V, 2008). El posicionamiento comprende el enfoque de la organización en cuanto a su forma de competir. (Porter, 1991). El éxito suele ser fruto de la preparación previa, realizada en la mayoría de los casos con gran esfuerzo personal. (Robles E, 2012). Los objetivos a corto plazo generan una ventaja competitiva, la empresa se vuelve más productiva. (Vega, 2014). Los objetivos de la empresa son metas que la entidad quiere alcanzar y que ayuden a lograr la misión de la empresa. (Jáuregui, 2017).

La ventaja competitiva, son las características propias de una organización y son aquellas actividades que contribuyen a la posición de costo relativo y crean una base para la diferenciación, (Benavides, 2002).

\section{OBJETIVO}

Definir cómo la participación en ferias comerciales de las empresas asociadas en Pro Olivo se relaciona con lacapacidad de negociación.

\section{METODOLOGÍA}

La investigación planteada es descriptiva, de nivel correlacional. Las unidades están compuestas por los gerentes y/o propietarios de las empresas asociadas a Pro Olivo y personal directivo de las oficinas a cargo de actividades de promoción de exportaciones que operan en Tacna y que financian actividades como ferias y misiones técnicas - comerciales. La población está compuesta por 30 empresas asociadas a Pro Olivo, de la ciudad de Tacna. Se diseñó un conjunto de preguntas estructuradas y las cuales se organizaron sistemáticamente en un orden lógico de manera que facilitará la comprensión del entrevistado y la labor del entrevistador. (Tabla 1)

\begin{tabular}{|c|c|c|}
\hline VARIABLE & INDICADOR & ÍTEM \\
\hline \multirow{3}{*}{$\begin{array}{l}\text { V1: Participación en } \\
\text { ferias comerciales }\end{array}$} & $\begin{array}{l}\text { Promoción del } \\
\text { producto }\end{array}$ & $\begin{array}{l}\text { Ideas para crear nuevos productos o servicios } \\
\text { Existen condiciones para lanzar nuevos productos } \\
\text { Posibilidad que las empresas puedan hacer Benchmarking } \\
\text { Se puede acceder a innovaciones o avances tecnológicos }\end{array}$ \\
\hline & $\begin{array}{l}\text { Distribución de } \\
\text { productos }\end{array}$ & $\begin{array}{l}\text { Información sobre análisis funcionamiento del mercado. } \\
\text { Alianzas con empresas del mismo sector } \\
\text { Se Toman decisiones sobre sistemas de distribución }\end{array}$ \\
\hline & $\begin{array}{l}\text { Variedad y calidad } \\
\text { de producto }\end{array}$ & $\begin{array}{l}\text { Revisión de productos de la competencia } \\
\text { Los feriantes analizan a su competencia directa } \\
\text { Los feriantes evalúan el nivel de servicio de la competencia } \\
\text { Se encuentra en las ferias ideas sobre servicios adicionales }\end{array}$ \\
\hline \multirow{3}{*}{$\begin{array}{l}\text { V2: Desempeño de } \\
\text { empresas asociadas }\end{array}$} & $\begin{array}{l}\text { Capacidad de } \\
\text { Negociación }\end{array}$ & $\begin{array}{l}\text { Se cuenta con capacidad de negociación necesaria } \\
\text { El personal cumple las funciones asignadas } \\
\text { Considera adecuadas las estrategias de mercadeo }\end{array}$ \\
\hline & Nivel Competitivo & $\begin{array}{l}\text { Las empresas de las ferias tienen una cartera creciente } \\
\text { Se cumple con las actividades programadas eficientemente } \\
\text { Las empresas satisfacen las necesidades de sus clientes } \\
\text { Los feriantes son organizaciones en acumulación }\end{array}$ \\
\hline & Logro de Objetivos & $\begin{array}{l}\text { Los feriantes generalmente alcanzan sus objetivos } \\
\text { Los reclamos se resuelven y atienden con amabilidad y rapidez } \\
\text { El futuro de los feriantes es auspicioso y alentador } \\
\text { Los logros obtenidos son de acuerdo a lo programado }\end{array}$ \\
\hline
\end{tabular}

\section{RESULTADOS}

En el trabajo de campo se efectúo las encuestas, utilizando como instrumento un cuestionario con 11 preguntas cada uno, que se efectuó a las diferentes empresas asociadas a Pro Olivo de la ciudad de 
Tacna, con la finalidad de poder evaluar las variables: variable 1, Participación en ferias comerciales y la variable 2 desempeño de empresas asociadas; con la finalidad de efectuar un análisis e interpretación de las respuestas obtenidas y poder contrastar las hipótesis posteriormente, a través de un cuestionario de 11 preguntas por cada variable a las empresas asociadas de Pro Olivo de la ciudad de Tacna, periodo 2016 - 2017.

Tabla 2

Percepción de desempeño y participación en ferias comerciales

\begin{tabular}{|c|c|c|}
\hline \multicolumn{3}{|c|}{ Ideas para crear nuevos productos o servicios } \\
\hline Casi nunca & 9 & $30 \%$ \\
\hline Algunas veces & 12 & $40 \%$ \\
\hline Casi siempre & 7 & $23 \%$ \\
\hline Siempre & 2 & $7 \%$ \\
\hline \multicolumn{3}{|c|}{ Existen condiciones para lanzar nuevos productos } \\
\hline Casi nunca & 4 & $14 \%$ \\
\hline Algunas veces & 10 & $33 \%$ \\
\hline Casi siempre & 16 & $53 \%$ \\
\hline \multicolumn{3}{|c|}{ Posibilidad que las empresas puedan hacer Benchmarking } \\
\hline Casi nunca & 6 & $20 \%$ \\
\hline Algunas veces & 9 & $30 \%$ \\
\hline Casi siempre & 15 & $50 \%$ \\
\hline \multicolumn{3}{|c|}{ Se puede acceder a innovaciones o avances tecnológicos } \\
\hline Casi nunca & 11 & $37 \%$ \\
\hline Algunas veces & 10 & $33 \%$ \\
\hline Casi siempre & 8 & $27 \%$ \\
\hline Siempre & 1 & $3 \%$ \\
\hline \multicolumn{3}{|c|}{ Información sobre análisis funcionamiento del mercado } \\
\hline Casi nunca & 4 & $13 \%$ \\
\hline Algunas veces & 20 & $67 \%$ \\
\hline Casi siempre & 5 & $17 \%$ \\
\hline Siempre & 1 & $3 \%$ \\
\hline \multicolumn{3}{|c|}{ Alianzas con empresas del mismo sector } \\
\hline Casi nunca & 8 & $27 \%$ \\
\hline Algunas veces & 14 & $46 \%$ \\
\hline Casi siempre & 8 & $27 \%$ \\
\hline \multicolumn{3}{|c|}{ Se Toman decisiones sobre sistemas de distribución } \\
\hline Nunca & 2 & $7 \%$ \\
\hline Casi nunca & 4 & $13 \%$ \\
\hline Algunas veces & 10 & $34 \%$ \\
\hline Casi siempre & 13 & $43 \%$ \\
\hline Siempre & 1 & $3 \%$ \\
\hline \multicolumn{3}{|c|}{ Revisión de productos de la competencia } \\
\hline Casi nunca & 14 & $47 \%$ \\
\hline Algunas veces & 7 & $23 \%$ \\
\hline Casi siempre & 9 & $30 \%$ \\
\hline \multicolumn{3}{|c|}{ Los feriantes analizan a su competencia directa } \\
\hline Nunca & 4 & $13 \%$ \\
\hline Algunas veces & 14 & $47 \%$ \\
\hline Casi siempre & 12 & $40 \%$ \\
\hline \multicolumn{3}{|c|}{ Los feriantes evalúan el nivel de servicio de la competencia } \\
\hline Nunca & 2 & $7 \%$ \\
\hline Casi nunca & 1 & $3 \%$ \\
\hline Algunas veces & 19 & $63 \%$ \\
\hline Casi siempre & 7 & $24 \%$ \\
\hline Siempre & 1 & $3 \%$ \\
\hline \multicolumn{3}{|c|}{ Se encuentra en las ferias ideas sobre servicios adicionales } \\
\hline Casi nunca & 5 & $17 \%$ \\
\hline Algunas veces & 15 & $50 \%$ \\
\hline Casi siempre & 10 & $33 \%$ \\
\hline \multicolumn{3}{|c|}{ Se cuenta con capacidad de negociación necesaria } \\
\hline Casi nunca & 5 & $17 \%$ \\
\hline Algunas veces & 10 & $33 \%$ \\
\hline Casi siempre & 14 & $47 \%$ \\
\hline Siempre & 1 & $3 \%$ \\
\hline \multicolumn{3}{|c|}{ El personal cumple las funciones asignadas } \\
\hline Casi nunca & 5 & $17 \%$ \\
\hline Algunas veces & 10 & $33 \%$ \\
\hline Casi siempre & 14 & $47 \%$ \\
\hline Siempre & 1 & $3 \%$ \\
\hline
\end{tabular}




\begin{tabular}{|c|c|c|}
\hline \multicolumn{3}{|c|}{ Considera adecuadas las estrategias de mercadeo } \\
\hline Casi nunca & 4 & $13 \%$ \\
\hline Algunas veces & 13 & $43 \%$ \\
\hline Casi siempre & 11 & $37 \%$ \\
\hline Siempre & 2 & $7 \%$ \\
\hline \multicolumn{3}{|c|}{ Las empresas de las ferias tienen una cartera creciente } \\
\hline Casi nunca & 3 & $10 \%$ \\
\hline Algunas veces & 15 & $50 \%$ \\
\hline Casi siempre & 11 & $37 \%$ \\
\hline Siempre & 1 & $3 \%$ \\
\hline \multicolumn{3}{|c|}{ Se cumple con las actividades programadas eficientemente } \\
\hline Casi nunca & 2 & $7 \%$ \\
\hline Algunas veces & 21 & $70 \%$ \\
\hline Casi siempre & 6 & $20 \%$ \\
\hline Siempre & 1 & $3 \%$ \\
\hline \multicolumn{3}{|c|}{ Las empresas satisfacen las necesidades de sus clientes } \\
\hline Casi nunca & 4 & $13 \%$ \\
\hline Algunas veces & 14 & $47 \%$ \\
\hline Casi siempre & 10 & $33 \%$ \\
\hline Siempre & 2 & $7 \%$ \\
\hline \multicolumn{3}{|c|}{ Los feriantes son organizaciones en acumulación } \\
\hline Casi nunca & 6 & $20 \%$ \\
\hline Algunas veces & 11 & $37 \%$ \\
\hline Casi siempre & 13 & $43 \%$ \\
\hline \multicolumn{3}{|c|}{ Los feriantes generalmente alcanzan sus objetivos } \\
\hline Casi nunca & 2 & $7 \%$ \\
\hline Algunas veces & 15 & $50 \%$ \\
\hline Casi siempre & 12 & $40 \%$ \\
\hline Siempre & 1 & $3 \%$ \\
\hline \multicolumn{3}{|c|}{ Los reclamos se resuelven y atienden con amabilidad y rapidez } \\
\hline Algunas veces & 16 & $53 \%$ \\
\hline Casi siempre & 12 & $40 \%$ \\
\hline Siempre & 2 & $7 \%$ \\
\hline \multicolumn{3}{|c|}{ El futuro de los feriantes es auspicioso y alentador } \\
\hline Nunca & 4 & $13 \%$ \\
\hline Algunas veces & 9 & $30 \%$ \\
\hline Casi siempre & 17 & $57 \%$ \\
\hline \multicolumn{3}{|c|}{ Los logros obtenidos son de acuerdo a lo programado } \\
\hline Casi nunca & 6 & $20 \%$ \\
\hline Algunas veces & 13 & $43 \%$ \\
\hline Casi siempre & 8 & $27 \%$ \\
\hline Siempre & 3 & $10 \%$ \\
\hline Total & 30 & $100 \%$ \\
\hline
\end{tabular}

En el análisis de las respuestas observadas en la tabla 2, se puede entender que falta implementar nuevas ideas y productos o servicios en las empresas asociadas a Pro Olivo para su participación en las diferentes ferias comerciales. Asimismo, se deduce que en su mayoría si existen las condiciones para lanzar nuevos productos y servicios, debiendo efectuar los correctivos necesarios para mejorar las condiciones. Muchas veces no se accede a innovaciones o avances tecnológicos en las ferias comerciales en la que se ha participado. Se observa que no es posible efectuar el análisis de la estructura y funcionamiento del mercado con la información obtenida en la feria, debiendo implementar nuevos procedimientos para mejorar el análisis de mercado.

Falta mejorar las acciones para poder crear alianzas entre las empresas participantes en las ferias comerciales, mejorar la participación de las empresas en la toma de decisiones sobre sistemas y formas de distribución, revisar la demanda de los productos o servicios de las empresas de la competencia, analizar mejor a la competencia acorde al levantamiento de información en torno a sus estrategias, mejorar el nivel de apreciación del servicio y las condiciones de pago de las empresas de la competencia. Mejorar sobre servicios adicionales que brindan las empresas participantes, mejorar la capacidad de negociación para ofertar sus productos por parte de las empresas que participan en las ferias comerciales, efectuar las charlas necesarias al personal de tal manera que permitan cumplir con responsabilidad el cumplimiento de sus funciones, implementar las estrategias necesarias que permitan obtener una buena aceptación del mercado, mejorar para contar con una cartera de clientes 
de manera creciente, debiendo establecer los procedimientos necesarios para su implementación, mejorar el cumplimiento de actividades programadas, debiendo establecer los correctivos necesarios, conocer las necesidades de sus clientes, debiendo efectuar la capacitación necesaria para una mayor atención en ello. Se debe mejorar en alcanzar los objetivos planeados, capacitar al personal en atención al público a fin de que haya una mayor satisfacción por parte de los clientes, relación a los reclamos que estos presentan, implementar procedimientos que permitan que la participación de las empresas feriantes sea más auspiciador y alentador. Los logros se vienen obteniendo de manera parcial, debiendo mejorar los procedimientos para un adecuado logro en función a la programación efectuada.

Existe relación directa entre la participación en ferias comerciales, de las empresas asociadas en Pro Olivo y su desempeño, periodo 2016 - 2017 (Tabla 3)

Tabla 3

Participación y desempeño de empresas asociadas

\begin{tabular}{|c|c|c|c|c|}
\hline & & & $\begin{array}{l}\text { Participación en } \\
\text { ferias comerciales }\end{array}$ & $\begin{array}{l}\text { Desempeño de } \\
\text { empresas asociadas }\end{array}$ \\
\hline \multirow{4}{*}{ Rho de Spearman } & \multirow{2}{*}{$\begin{array}{l}\text { Participación en } \\
\text { ferias comerciales }\end{array}$} & Coeficiente de correlación & 1,000 & ,510 \\
\hline & & Sig. (bilateral) & . &, 007 \\
\hline & \multirow{2}{*}{$\begin{array}{l}\text { Desempeño de } \\
\text { empresas asociadas }\end{array}$} & Coeficiente de correlación & ,510 & 1,000 \\
\hline & & Sig. (bilateral) &, 007 & . \\
\hline
\end{tabular}

Asimismo, los resultados, indican que existe relación directa entre la participación en ferias comerciales y su capacidad de negociación ( $p$ : 0.001), nivel competitivo ( $p: 0.010)$ y el logro de sus objetivos (p:0.001).

\section{DISCUSIÓN}

Del análisis realizado se puede comprobar, a lo largo de este estudio que efectivamente la relación entre la participación en ferias comerciales de las empresas asociadas en Pro Olivo y su desempeño es directa. Asimismo, se observa que falta implementar nuevas ideas y productos o servicios en las empresas asociadas a Pro Olivo para su participación en las diferentes ferias comerciales. En lo que se refiere a si es válido encontrar en una feria comercial ideas para crear nuevos productos o servicios, el $40 \%$ manifiesta que algunas veces, el $30 \%$ contesto que casi nunca, el 23\% indicó que casi siempre y el $7 \%$ manifestó que siempre. En la pregunta si existen las condiciones y las oportunidades para lanzar nuevos productos y servicios, el 53\% manifiesta que casi siempre, el 33\% contesto que algunas veces, el $14 \%$ manifestó que casi nunca. De lo que se puede entender que en su mayoría si existen las condiciones para lanzar nuevos productos y servicios, debiendo efectuar los correctivos necesarios para mejorar las condiciones. En lo relacionado a si es posible que en las ferias se puedan acceder a innovaciones o avances tecnológicos; el 37\% manifiesta que casi nunca, el 33\% contestó que algunas veces, el $27 \%$ indicó que casi siempre, el $3 \%$ contestó que siempre; de lo que se entiende que muchas veces no se accede a innovaciones o avances tecnológicos en las ferias comerciales en la que se ha participado. En lo relacionado a si en las ferias comerciales es posible para las empresas revisar la demanda de los productos o servicios de las empresas de la competencia; el $47 \%$ indica que casi nunca, el $30 \%$ manifiesta que casi siempre, el $23 \%$ indica que algunas veces, de lo que puede entender que en algunos casos si se puede revisar la demanda de los productos o servicios de las empresas de la competencia. Niño L. (2014), concluye que las ferias y exposiciones son reconocidas como escenarios en donde empresas y personas interactúan buscando satisfacer necesidades de negocio, conocimiento, esparcimiento, comunicación e interacción, mediante el intercambio de bienes, servicios e información. Mediante esta dinámica, permiten la construcción de redes de relaciones no sólo de negocio sino también sociales y son en mayor parte las ferias especializadas, las que crean el ambiente adecuado para que se gesten comunidades con estos intereses (profesionales y personales). 
Las ferias y exposiciones son los espacios propicios y adecuados para que las organizaciones interactúen con sus clientes potenciales. También, Olivares P. (2014), concluye que las ferias internacionales son una herramienta de promoción ampliamente utilizada alrededor del mundo. Tanto en Europa como en Estados Unidos, las ferias son eventos de carácter relevante en términos de lanzamiento de nuevos productos, presentaciones, demostraciones, ventas o posicionamiento de marcas, ya que permiten la interacción directa entre oferentes y consumidores. Mauricio J. (2010), concluye que la adopción de la participación en las ferias como estrategia de comercio y promoción de exportaciones, es función directa del empresario exportador, lógicamente contando con el compromiso y dedicación del proceso de asesoría comercial.

Los propietarios de las empresas asociadas a Pro Olivo deberán implementar nuevas ideas y productos o servicios para mejorar su participación en las diferentes ferias comerciales, implementar la capacidad de negociación para ofertar sus productos por parte de las empresas que participan en las ferias comerciales. Asimismo, deberán implementar las condiciones necesarias para el lanzamiento de nuevos productos y servicios, implementar procedimientos que permitan revisar la demanda de los productos o servicios de las empresas de la competencia, e implementar la capacitación oportuna que permita alcanzar los objetivos planeados, y mejorar su participación, obteniendo el logro deseado en función a la programación efectuada.

\section{REFERENCIAS BIBLIOGRÁFICAS}

Altuve C (1999) Relación entre estilos de liderazgo según el modelo Bolman y Deal y el desempeño laboral. P, 36.

Bengochea, (2008) Concepto de competitividad empresarial. [Sitio Web] Recuperado de: https://www.gestiopolis.com/concepto-competitividad-empresarial/

Bernal (2018) Manual para organizadores de ferias. Recuperado de: https://pascualbernal.wordpress.com/manual-para-organizadores-de-ferias/

Caferri (2017) Seis pasos para mejorar tu capacidad de negociación. [Sitio Web] Recuperado de: https://www.aboutespanol.com/seis-pasos-para-mejorar-tu-capacidad-de-negociacion2480074

Cavero U. (2018) Pro Olivo [Sitio Web]. Recuperado: http://proolivo.com/empresa

Estrada (2010) Las ferias de comercio como herramienta de mercadeo para la promoción de exportaciones . [Tesis de pregrado] Universidad Tecnológica Equinoccial. Quito. Ecuador.

Hernández M. (2006) Qué es negociación, tipos, etapas y técnicas efectivas

Jáuregui (2017) Objetivos de una empresa: tipos y sus características. [Sitio Web] Recuperado de: https://www.lifeder.com/objetivos-empresa/

Jiménez A. (2015), Organización de eventos de marketing y comunicación [Sitio Web] https://books.google.com.pe

Lamb H. (2006) Canales de Distribución - Canales de Distribución y Logística.

Lamb, Hair, McDaniel, Fundamentos de marketing.

Mauricio J. (2010 Las Ferias De Comercio Como Herramienta De Mercadeo Para La Promoción De Exportaciones . [Tesis de pregrado] Universidad Tecnológica Equinoccial. Bogotá.

Niño L. (2014) Participación En Ferias Y Exposiciones Para Incrementar Los Contactos De Negocio

Niño L. (2014), Participación en ferias y exposiciones para incrementar los contactos de negocio. [Tesis de pregrado] Universidad Militar Nueva Granada.

Olivares P. (2014), Las Ferias Internacionales Patrocinadas por Pro Chile: ¿Cuál es la Percepción de las Empresas Participantes? . [Tesis de pregrado] Universidad de Chile.

Philip K. \& Keller, K. (2012), Dirección de Marketing- decimocuarta Edición - México

Philip K., armstrong Gary (2003) Fundamentos De Marketing, México, Pearson Prentice Hall, 6a. Edición.

Philip K., Fundamentos de marketing. 
PhilipK. (2017) canales de distribución - Fundamentos de marketing.

Pro Olivo, (2017) Perú: Pro Olivo crea un grupo gestor para impulsar la innovación dentro del sector olivarero. [Sitio Web] Recuperado de: http://ecomercioagrario.com/peru-pro-olivo-crea-ungrupo-gestor-para-impulsar-la-innovacion-dentro-del-sector-olivarero/

Puchalt J. \& Munuera J. (2008), Panorama Internacional De Las Ferias Comerciales

Reales H. (2003), Características del producto.

Reyes (2009) Estrategias de comunicación de tres ferias exitosas realizadas en corferias (expoartesanias, feria internacional del libro de Bogotá, international footwear \& leather show ifls) , [Tesis de pregrado] Pontifica Universidad Javeriana. Bogotá.

Robles E. (2012) Motivación para el logro de los objetivos.

Román, (2016) Caracterización de la capacitación y competitividad en las mype de servicio rubro pizzería en el centro de Piura, año 2016. [Tesis de pregrado] Universidad Católica Los Ángeles Chimbote.

Sánchez A. (2017) Promoción Según Los Expertos

Sarmiento García Manuel (1995), El Mercado De Ferias Y Exposiciones Y Otros Viajes De Negocios.

Stanton, E y Walker, MI (2010), Fundamentos de Marketing

Stern, L. (2015), Canales de Comercialización

Thompson I. (2010), Estrategia \& Marketing

Valderrama, W. (2011) Las manifestaciones promocionales como estrategia de Marketing, Tacna Perú, Fondo Editorial de la Universidad Privada de Tacna.

Valietti B (2008) Concepto de competitividad empresarial .

Vega (2014) Tacna exportará 20 mil toneladas de olivos. [Sitio Web] Recuperado de: https://diariocorreo.pe/peru/tacna-exportara-20-mil-toneladas-de-olivos-211547/

Velázquez E (2012), Canales de Distribución y Logística. 